\title{
Electrochemical and optical properties of a new donor-acceptor type conjugated polymer derived from 3,4-didodecyloxythiophene
}

\author{
PRAMOD KUMAR HEGDE, A VASUDEVA ADHIKARI* and M G MANJUNATHA \\ Department of Chemistry, National Institute of Technology, Surathkal, Srinivasnagar 575 025, India
}

MS received 8 May 2009; revised 25 November 2009

\begin{abstract}
In this communication, we report the synthesis and characterization of a new donor-acceptor type conjugated polymer carrying alternate 3,4-didodecyloxythiophene and (1,3,4-oxadiazol-yl)pyridine moieties and evaluation of its optical and electrochemical properties. The polymer was synthesized through precursor polyhydrazide route. It has well defined structure, stability and it shows good solubility in common organic solvents. Optical and electrochemical properties were studied by UV-visible, fluorescence spectroscopy and cyclic voltammetric studies, respectively. It displays bluish-green fluorescence both in solution and in film state. Cyclic voltammetric studies showed that the polymer P1 possesses a HOMO energy level of $-6 \cdot 01$ and LUMO energy level of $-\mathbf{3 . 3 4} \mathrm{eV}$. The preliminary studies clearly reveal that the new polymer can have potential application in the fabrication of light emitting diodes. The studies on its device application are in progress.
\end{abstract}

Keywords. Conjugated polymers; (1,3,4-oxadiazol-yl)pyridine; electrochemical properties; fluorescence; light emitting diodes.

\section{Introduction}

Recently a great deal of attention was focused on conducting polymers due to their extensive applications in different electronic and optoelectronic devices. The design and development of conjugated soluble polymers is presently the subject of vast research interest. These materials have the advantage of facile colour tunability, good film forming properties, solubility, and adequate mechanical properties in comparison with their inorganic counterparts. Also, polymers have distinct advantages such as higher stability, processibility and film forming ability over low molecular weight organic compounds. In this decade, several conjugated polymers have been developed; amongst them poly (1,4-phenylenevinylene) (PPV) (Burroughes et al 1990), poly( $p$-phenylene) (PPs) (Grem et al 1992), polyfluorenes (PFs) (Leclerc 2001) and polythiophenes (PTs) were in the focus of investigations. Much attention was concentrated on conjugated polymers due to the broad range of applications for which they are potentially useful (Skotheim and Reynolds 1998). Photovoltaic devices (Brabec et al 2001), light emitting diodes (Friend et al 1999), field effect transistors (Stutzmann et al 2003), electrochromic devices (Argun et al 2003), and various types of sensors (McQuade et al 2000) based on conjugated polymers are under investigation by numerous researchers around the world. A polymer that possesses a

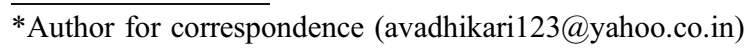

combination of several of these properties is especially an attractive target (Pei et al 2000; Thompson et al 2005).

In the present work, it has been planned to synthesize a new fluorescent $p$ - and $n$-dopable polymer containing 3,4dialkoxythiophene as $p$-type segment and 1,3,4-oxadiazolyl pyridine moiety as $n$-type segment in the polymer chain. In our new synthetic design, it has been predicted that the presence of pyridine rings reduces the steric repulsion between the bulky alkoxy-thiophene groups and increases the planarity of the polymer chain that could help to reduce the bandgap. Further, presence of electron releasing didodecyloxy groups and electron withdrawing oxadiazole and pyridine moieties in the polymer backbone is anticipated to induce greater delocalization of $\pi$-electrons along the polymer chain and hence it is expected to show better fluorescence efficiency ( $\mathrm{Li}$ et al 2004). Also, the polymer has been predicted to show improved solubility owing to the presence of pyridine moiety and alkoxy substitution on the thiophene ring of the polymer chain.

\section{Experimental}

\subsection{Materials and instrumentation}

All the solvents and reagents were of analytical grade, used without further purification. Infrared spectra of intermediate compounds and the polymer were recorded on a Nicolet Avatar 5700 FTIR spectrophotometer. The UV-visible and fluorescence spectra were taken in GBC Cintra 101 UV- 
visible and Perkin Elmer LS55 fluorescence spectrophotometers, respectively. ${ }^{1} \mathrm{H}-\mathrm{NMR}$ spectra were obtained with AMX-400 MHz FT-NMR spectrometer using TMS as internal reference. Elemental analyses were performed on a Flash EA1112 CHNS analyser. The photoluminescence quantum efficiency of the polymer film was measured by HORIBA Jobin-Yvon quantum yield calculator. The electrochemical study was carried out using a AUTOLAB PGSTAT30 electrochemical analyser. Molecular weight was determined with Water's make Gel permeation chromatograph. The thermal stability of the polymer was studied by SII EXSTAR6000-TG/DTA6300 thermogravimetric analyzer. The required 3,4-didodecyloxythiophene-2,5-dicarbohydrazide (c) was synthesized according to the reported procedure (Udayakumar and Adhikari 2006).

\subsection{Synthesis of polymer, $P 1$}

The polymer, P1 was synthesized from 3,4-didodecyloxythiophene 2,5-carboxylate following the reaction sequence as described in scheme 1.

\subsection{Preparation of polyhydrazide, $\mathrm{PH}$}

To a mixture of 1 equivalent of 3,4-didodecyloxythiophene 2,5-dicarbohydrazides (c), 2 equivalents of anhydrous aluminum chloride and $0 \cdot 1 \mathrm{~mL}$ of pyridine, 1 equivalent of pyridine 2,6-diacid chloride (d) was added slowly at room temperature with stirring which was continued for $5 \mathrm{~h}$. Further, it was heated at $80^{\circ} \mathrm{C}$ for $20 \mathrm{~h}$. After cooling to room temperature the reaction mixture was poured into ice cold water and the precipitate separated was collected by filtration. It was washed with water followed by acetone and finally dried in vacuum to get the polyhydrazide, $\mathrm{PH}$.

Yield: $85 \%$, IR: $3311 \mathrm{~cm}^{-1}(\mathrm{~N}-\mathrm{H}), 1714 \mathrm{~cm}^{-1}(\mathrm{C}=\mathrm{O})$, elemental: $\left(\mathrm{C}_{37} \mathrm{H}_{57} \mathrm{~N}_{5} \mathrm{O}_{6} \mathrm{~S}\right)$, calc, C, 63.49; H, 8.21; N, 10.10; S, 4.58; found : C, 63.20; H, 8.48; N, 9.74; S, 4.60.

\subsection{Preparation of polymer, $P 1$}

A mixture of polyhydrazide $(\mathrm{H}, 0.5 \mathrm{~g})$ and $20 \mathrm{~mL}$ phosphorus oxychloride was heated at $100^{\circ} \mathrm{C}$ for $8 \mathrm{~h}$ with stirring. The reaction mixture was then cooled to room temperature and poured to excess of ice. The resulting precipitate was collected by filtration, washed with water followed by acetone and dried in vacuum oven to get the copolymer P1 in yield 85\%. FTIR: 2924, 2855, $2762 \mathrm{~cm}^{-1}$ (C-H stretching of aliphatic segments), $1594(>\mathrm{C}=\mathrm{N}-$ of 1,3,4-oxadiazole), $1460 \mathrm{~cm}^{-1}$ (aromatic), $1048 \mathrm{~cm}^{-1}$ (=C-O$\mathrm{C}=$ stretching of oxadiazole). Elemental: $\left(\mathrm{C}_{37} \mathrm{H}_{53} \mathrm{~N}_{5} \mathrm{O}_{4} \mathrm{~S}\right)$, calc: C, 66.94; H, 8.05; N, 10.55; S, 4.83, found : C, 66.80; H, $8.28 ; \mathrm{N}, 10 \cdot 50 ; \mathrm{S}, 4.90,{ }^{1} \mathrm{H}$ NMR: $\left(\mathrm{CDCl}_{3} \delta, \mathrm{ppm}\right): 0.86$ $\left(t, 6 \mathrm{H},-\mathrm{CH}_{3}\right), 1 \cdot 2-1.9\left(\mathrm{~m}, 40 \mathrm{H}\right.$, alkyl), $4.38\left(t, 4 \mathrm{H},-\mathrm{OCH}_{2}\right)$, $8 \cdot 1-8 \cdot 3\left(m, 3 \mathrm{H}\right.$, pyridine), molecular weight $\left(M_{\mathrm{w}}\right): 15985$.

\section{Results and discussion}

\subsection{Synthesis and characterization of polymer}

As given in scheme 1, the precursor polyhydrazide was synthesized by polycondensation of 3,4-didodecyloxythiophene-2,5-carbonyldihydrazide(c) with pyridine 2,6-dicarbonyl chloride(d) in presence of anhydrous aluminum chloride and pyridine. The polyhydrazide $(\mathrm{PH})$ was cyclized to polyoxadiazole (P1) using phosphorus oxychloride. The formation of precursor polyhydrazide was evidenced by its FTIR spectral and elemental analyses. The FTIR spectrum of polyhydrazide exhibited sharp peaks at 3311 and 1714 $\mathrm{cm}^{-1}$ accounting for $>\mathrm{N}-\mathrm{H}$ and $>\mathrm{C}=\mathrm{O}$ groups, respectively as shown in figure 1 . The successful conversion of polyhydrazide into polyoxadiazole (P1) was confirmed by its FTIR spectrum. In its IR spectrum, disappearance of $>\mathrm{C}=\mathrm{O}$ and $>\mathrm{N}-\mathrm{H}$ stretching absorption bands and appearance of a sharp peak at around $1594 \mathrm{~cm}^{-1}$ due to imine of the oxadiazole ring indicate the cyclization (figure 1).<smiles>CCOC(=O)c1sc(C(=O)OCC)c(O)c1O</smiles>

a

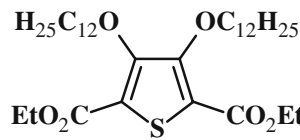

b

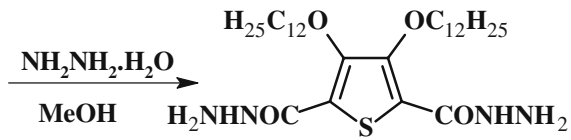

c<smiles>CCCOc1c(C(=O)NNC(=O)c2cccc(C(C)(C)C)n2)sc(C(=O)NNC(=O)C(C)(C)C)c1OCCC</smiles>

Scheme 1. Synthetic route for the preparation of polymer P1. 


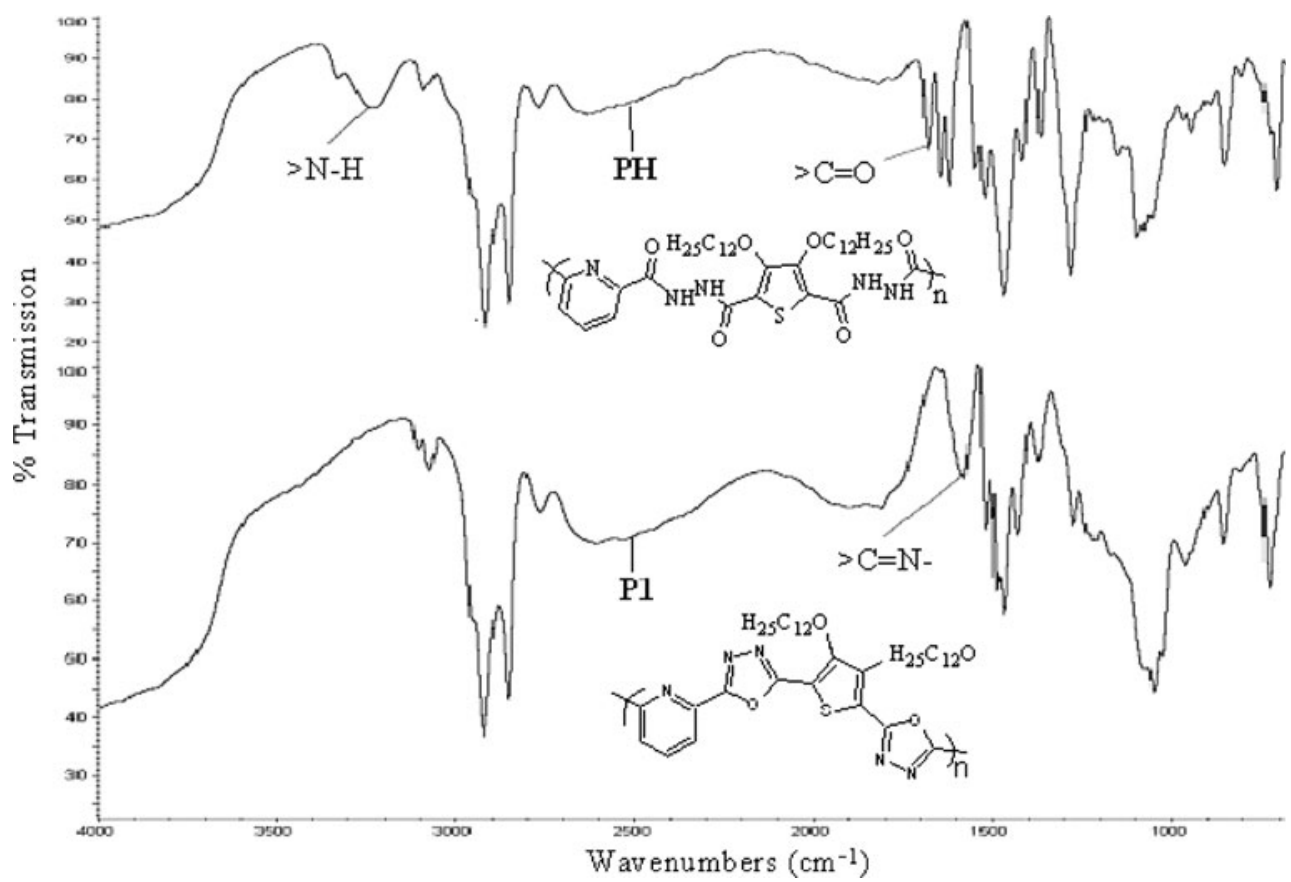

Figure 1. FTIR spectrum of polyhydrazide $(\mathrm{PH})$ and the polymer $\mathrm{P} 1$.

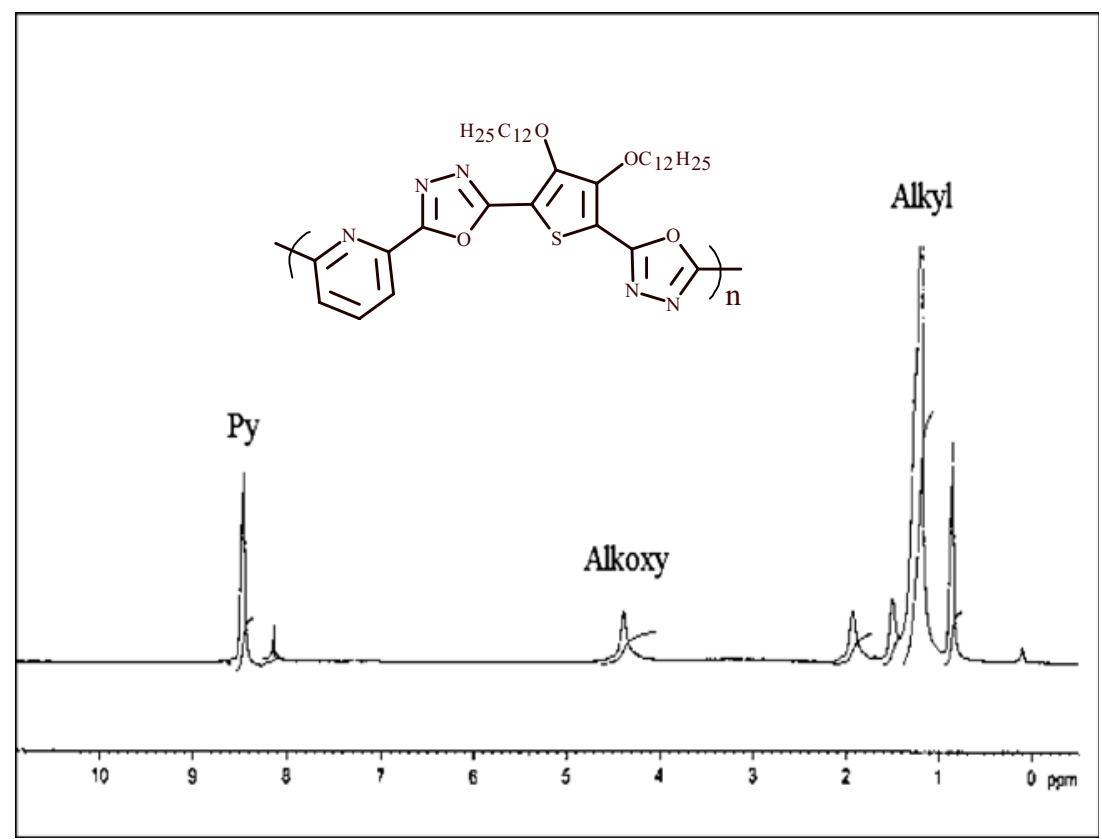

Figure 2. ${ }^{1} \mathrm{H}-\mathrm{NMR}$ spectrum of $\mathrm{P} 1$.

The chemical structure of P1 was further confirmed by its ${ }^{1} \mathrm{H}-\mathrm{NMR}$ spectroscopy and elemental analysis. The ${ }^{1} \mathrm{H}-\mathrm{NMR}$ spectra of copolymer P1 showed a multiplet at $\delta$ $8 \cdot 10-8 \cdot 33 \mathrm{ppm}$, due to the three protons of the pyridine (Py) ring (figure 2). Peaks corresponding to the protons of the alkoxy (- $\mathrm{OCH}_{2}-$, alkoxy) groups at 3 and 4-positions of the thiophene ring appeared at $\delta 4.38 \mathrm{ppm}$. A set of multiplet peaks which corresponds to $-\left(\mathrm{CH}_{2}\right)_{20}-$ (alkyl) appeared at $\delta$
$1 \cdot 20-1.90 \mathrm{ppm}$. The methyl protons $\left(-\mathrm{CH}_{3}\right)$ of the alkoxy substitution resonated as a triplet at $\delta 0.86 \mathrm{ppm}$. The results of elemental analysis of polymer were in agreement with its expected empirical formula. Its weight average molecular weight $\left(M_{\mathrm{w}}\right)$ was found to be 15985 . The polymer is found to be thermally stable up to about $320^{\circ} \mathrm{C}$. The thermogravimetric (TGA) trace of the polymer is given in figure 3. 


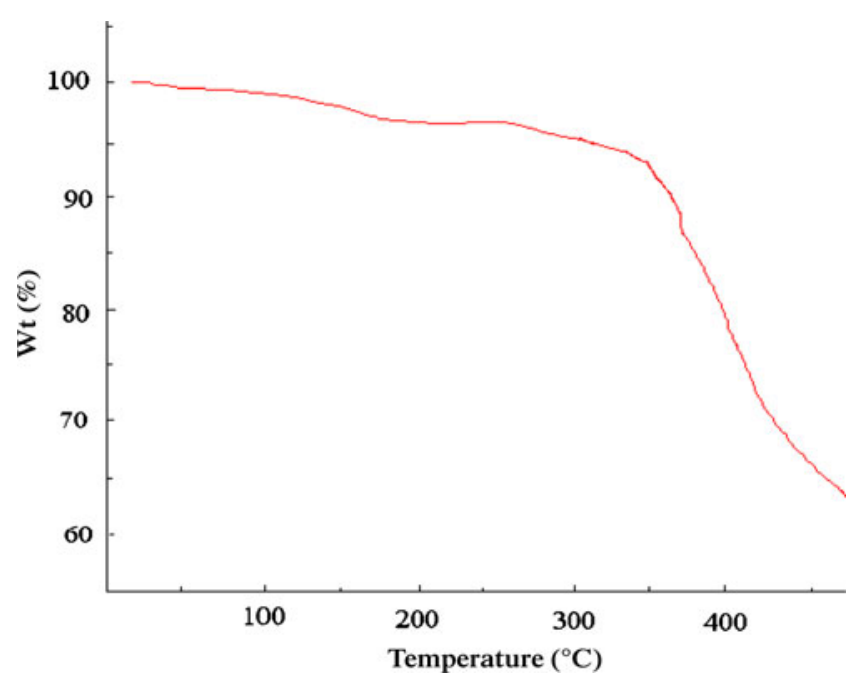

Figure 3. TGA trace of the polymer P1.

\subsection{Electrochemical studies}

Cyclic voltammetry (CV) was employed to determine redox potentials of new polymer and then to estimate the HOMO and LUMO, which is of importance to determine the bandgap. The cyclic voltammogram of the polymer coated on a glassy carbon electrode was measured using AUTOLAB PGSTAT-30 electrochemical analyser, using a $\mathrm{Pt}$ counter electrode and a $\mathrm{Ag} / \mathrm{AgCl}$ reference electrode, immersed in the electrolyte $\left[0 \cdot 1 \mathrm{M}(n-\mathrm{Bu})_{4} \mathrm{NClO}_{4}\right.$ in acetonitrile] at a scan rate of $25 \mathrm{mV} / \mathrm{S}$. Electrochemical data of P1 is summarized in table 1 .

In cathodic sweep, the polymer showed reduction peak at $-1.38 \mathrm{eV}$. This reduction potential is lower than that of 2-(4tirt-butylphenyl)-1,3,4-oxadiazole (PBD) (Strukelji et al 1995; Janietz et al 1997), which is one of the most widely used electron transporting materials. While in anodic sweep, it showed small oxidation peak at $+2 \cdot 02$ (figure 4), comparable with some donor acceptor type of polymers containing 1,3,4-oxadiazole moieties reported in the literature (Strukelji et al 1995; Janietz et al 1997). The onset oxidation and reduction potentials were used to estimate the highest occupied molecular orbital (HOMO) and lowest unoccupied molecular orbital (LUMO) energy levels of the copolymer P1. The equations reported in the literature (Yang and Jenekhe 1995; deLeeuw et al 1997), $E_{\mathrm{HOMO}}=$ $-\left[E_{\text {onset }}^{\text {oxd }}+4 \cdot 40 \mathrm{eV}\right)$ and $E_{\mathrm{LUMO}}=-\left[E_{\text {onset }}^{\mathrm{red}}-4 \cdot 40 \mathrm{eV}\right)$, where

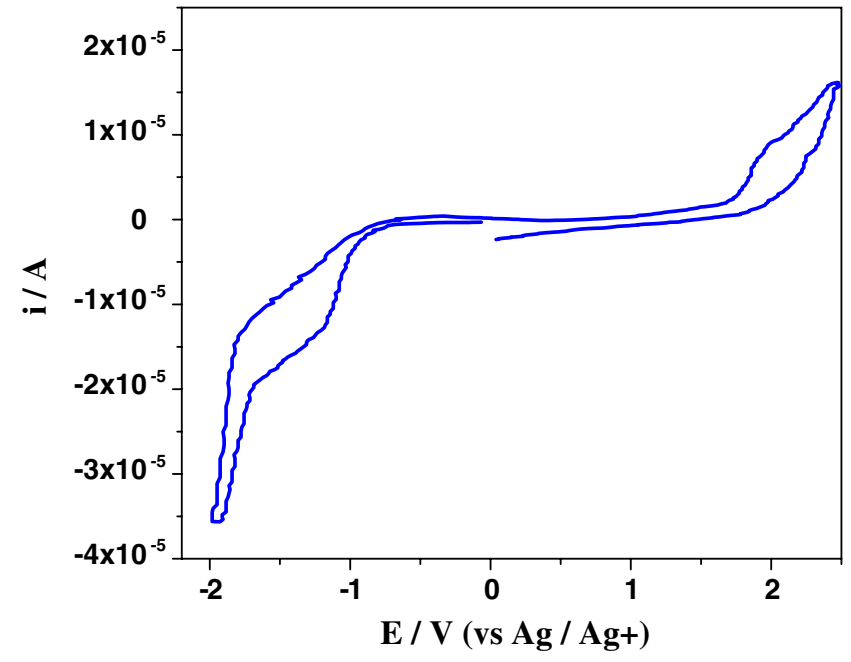

Figure 4. Oxidation and reduction cyclic voltammetric waves of the polymer $\mathrm{P} 1$.

$E_{\text {onset }}^{\text {oxd }}$ and $E_{\text {onset }}^{\text {red }}$ are the onset potentials vs SCE for the oxidation and reduction of this polymer, was referred for the calculations. Electrochemical potentials and energy levels of the polymer are tabulated in table 1. The HOMO energy level of the polymer is estimated to be -6.01 and the LUMO energy level is found to be $-3.34 \mathrm{eV}$. These values are lower than those of cyano-PPV $(-3.02)$ and some aromatic poly(oxadiazole)s $(-2.80$ to $-2.90 \mathrm{eV})$ (Bradley 1993; Carvini et al 1997), indicating that this polymer has a very good electron injecting ability. Its bandgap $\left(E_{\mathrm{g}}\right)$ is estimated to be $2.67 \mathrm{eV}$.

The energy barriers between the emitting polymers and electrodes can be estimated by comparing the work functions of the electrodes with HOMO and LUMO energy levels of emitting polymers. As reported in the literature, the hole-injection barrier, $\Delta E_{\mathrm{h}}$, is given by: $\Delta E_{\mathrm{h}}=E_{\mathrm{HOMO}}-4.8 \mathrm{eV}$, where $4.8 \mathrm{eV}$ is the work function of the ITO anode, while the electron-injection barrier $\Delta E_{\mathrm{e}}$, is given by: $\Delta E_{\mathrm{e}}=4 \cdot 3-E_{\mathrm{LuMO}} \mathrm{eV}$, where $4.3 \mathrm{eV}$ is the work function of aluminium cathode. The difference between the electron and hole-injection barriers ( $\Delta E_{\mathrm{e}}-\Delta E_{\mathrm{h}}$ ) is a useful parameter for evaluating the charge carrying ability. Generally lower value indicates better injection balance of electrons and holes from the cathode and anode, respectively. As shown in table 1, our polymer

Table 1. Electrochemical potentials, energy levels and energy barriers for electron/hole injection for the polymer P1.

\begin{tabular}{lccccccccccc}
\hline Polymer & $E_{\text {oxd }}^{\text {ons }}$ & $E_{\text {red }}^{\text {onse }}$ & $E_{\text {oxd }}$ & $E_{\text {red }}$ & $\begin{array}{l}\text { HOMO } \\
(\mathrm{eV})\end{array}$ & $\begin{array}{l}\text { LUMO } \\
(\mathrm{eV})\end{array}$ & $\begin{array}{l}\Delta E_{\mathrm{g}}{ }^{\mathrm{a}} \\
(\mathrm{eV})\end{array}$ & $\begin{array}{l}\Delta E_{\mathrm{h}}{ }^{\mathrm{b}} \\
(\mathrm{eV})\end{array}$ & $\begin{array}{l}\Delta E_{\mathrm{e}}{ }^{\mathrm{c}} \\
(\mathrm{eV})\end{array}$ & $\begin{array}{l}\Delta E_{\mathrm{e}}-\Delta E_{\mathrm{h}} \\
(\mathrm{eV})\end{array}$ \\
\hline $\mathrm{P} 1$ & 1.68 & -0.99 & 2.02 & -1.38 & -6.01 & -3.34 & 2.67 & 1.21 & 0.96 & 0.25 \\
\hline
\end{tabular}

${ }^{\mathrm{a}}$ Electrochemical bandgap, ${ }^{\mathrm{b}}$ energy barrier between HOMO and work function of ITO anode, ${ }^{\mathrm{c}}$ energy barrier between LUMO and work function of $\mathrm{Al}$ cathode. 
displayed $\Delta E_{\mathrm{e}}, 0.96 \mathrm{eV}$ which is lower than that of PPV $(1.8 \mathrm{eV})$ and some other $p$-type polymers. Further, the polymer exhibited higher value of $\Delta E_{\mathrm{h}}$, i.e. $1 \cdot 21 \mathrm{eV}$ which is higher than that of PPV $(0 \cdot 3 \mathrm{eV})$ and lower than that of PBD (Carvini 2007) and tris (8-hydroxyquinoline) aluminium $\left(\mathrm{Alq}_{3}\right)$ (Im et al 2001), which are widely used as electron transporting/hole blocking materials. In the polymer, the lower value of $\Delta E_{\mathrm{e}}$ is attributed to the presence of electron affinitive oxadiazole and pyridine moieties and the higher value of $\Delta E_{\mathrm{h}}$ is due to the occurrence of bulky didodecyl oxy substituted thiophene rings in polymer backbone. Further, the barrier energy difference $\left(\Delta E_{\mathrm{e}}-\right.$ $\Delta E_{\mathrm{h}}$ ) of the polymer was found to be $0.25 \mathrm{eV}$. This confirms that the polymer possesses an improved charge carrying property. These studies clearly indicate that newly synthesized donor-acceptor type conjugated polymer is a promising material for the development of efficient PLED.

\subsection{Optical properties}

Optical property of the polymer was studied by UV-vis and fluorescence spectroscopic studies. The UV-vis absorption and fluorescence emission spectra were recorded both in dilute DMF solution and thin film. The absorption maximum of the polymer in dilute solution is $342 \mathrm{~nm}$. The spectrum in thin film form $(350 \mathrm{~nm})$ showed red shift indicating the presence of inter-chain interactions in the solid state. The optical bandgap $\left(E_{\mathrm{g}}\right)$ was found to be $2.76 \mathrm{eV}$. It emits intense bluish green fluorescence emission peaks at $473 \mathrm{~nm}$ in its dilute solution. The UVvis and fluorescence spectrum of the polymers in dilute solution is shown in figures 5 and 6, respectively. A red shift of $10 \mathrm{~nm}$ has been observed. The fluorescence quantum yield (Davey et al 1995) of the polymer in solution was determined using quinine sulfate as standard

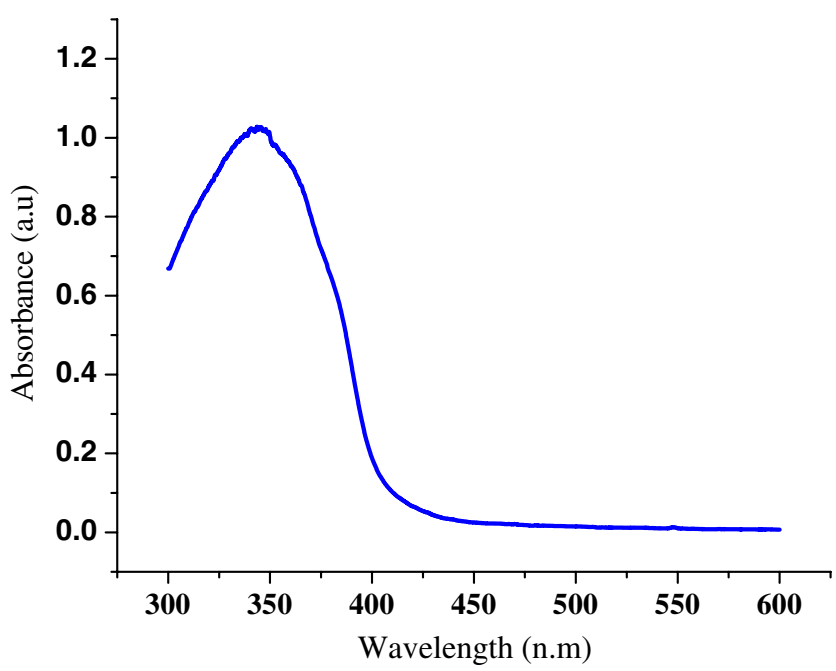

Figure 5. UV-vis spectrum of the polymer P1 in solution.

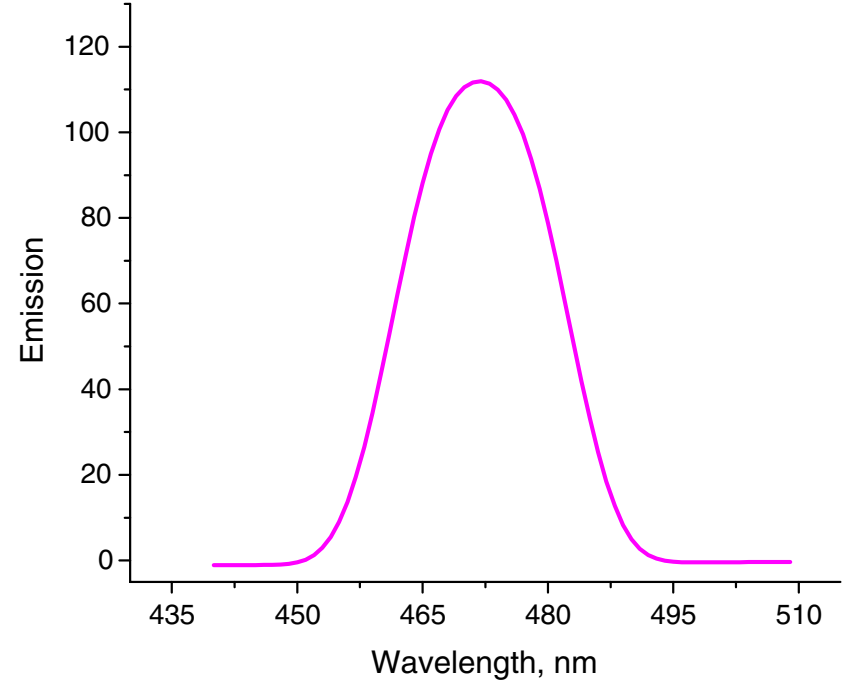

Figure 6. Fluorescence emission spectrum of the polymer P1 in solution.

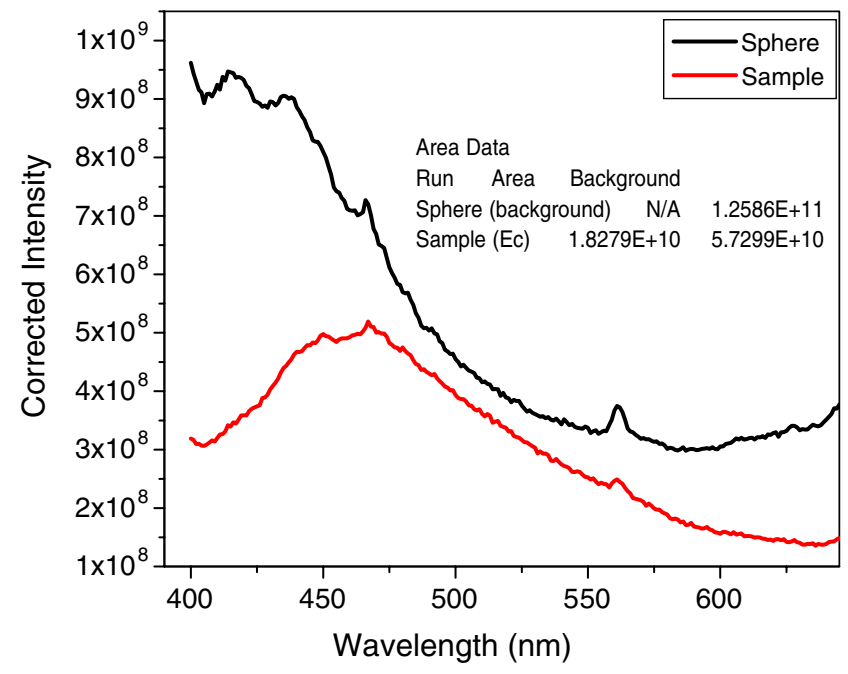

Figure 7. Fluorescence emission spectrum of the polymer P1 in thin film.

and it is found to be $32 \%$. These results indicate that the new polymer can be used as fluorescent light emitting material in devices. The detailed study on its PLED application is in progress.

Photoluminescence quantum efficiency of the polymer (PLQE) in solid state was determined using thin film of the polymer at the excitation wavelength of $342 \mathrm{~nm}$. At this wavelength the polymer film showed $46.4 \%$ absorption, 53.6\% transmission with an absorbance of 0.27 au. The quantum efficiency at this wavelength was calculated to be $69 \%$ (with an error of $10 \%$ ). Fluorescence emission spectrum of the polymer P1 in thin film is shown in figure 7 . 


\section{Conclusions}

In conclusion, a new donor-acceptor type conjugated polymer P1 with (3,4-didodecyloxythiophene) as donor and (1,3,4-oxadiazolyl)pyridine as acceptor moiety has been successfully synthesized and characterized by different spectroscopic techniques. It possesses a well defined structure, and displays good solubility and thermal stability. The electrochemical studies showed that the polymer possesses high-lying HOMO $(-6.01 \mathrm{eV})$ and low-lying LUMO $(-3.34 \mathrm{eV})$ energy levels because of the presence of alternative electron donor and electron acceptor units along the polymer backbone. The results of electrochemical studies confirm that the polymer possesses potential of charge carrying property. Also, its bandgap $\left(E_{\mathrm{g}}\right)$ was found to be $2.67 \mathrm{eV}$. The optical properties indicate that the polymer emits bluish-green fluorescence under the irradiation of UV-light and its quantum yield was estimated to be $32 \%$ and $69 \%$ in solution and film state, respectively. The results reveal that the new polymer possesses good intrinsic electronic properties comparable to or better than many other reported donor-acceptor type conjugated polymers. Therefore, it can be concluded that P1 can have potential application in PLED devices.

\section{Acknowledgements}

We are grateful to NMR Research Centre, Indian Institute of Science, Bangalore and Regional Research Laboratory, Trivandrum, for instrumental analyses.

\section{References}

Argun A A, Cirpan A and Reynolds J R 2003 Adv. Mater. 151338 Brabec C J, Sariciftci N S and Hummelen J C 2001 Adv. Funct. Mater. 1115

Bradley D D C 1993 Synth. Met. 544031

Burroughes J H et al 1990 Nature 347539

Carvini R 2007 Appl. Phys. Lett. 792131

Carvini R, Spencer G W C, Holmes A B, Moratti S C and Friend R 1997 Synth. Met. 84359

Davey A P, Elliott S, O'Conner O and Blau W J 1995 Chem. Soc. Chem. Commun. 141433 (doi:10.1039/C39950001433)

deLeeuw D M, Simenon M M J, Brown A B and Einerhand R E F 1997 Synth. Met. 8753

Friend R H et al 1999 Nature 121397

Grem G, Leditzky G, Ullrich B and Leising G 1992 Adv. Mater. 4 36

Im W-B, Hwang H-K, Lee L-G, Han K and Kim Y 2001 Appl. Phys. Lett. 791387

Janietz S, Wedel A and Friedrich R 1997 Synth. Met. 84381

Leclerc M J 2001 Polym. Sci. Part A: Polym. Chem. 392867

Li Najun et al 2004 Mater. Lett. 253115

McQuade D T, Pullen A E and Swager T M 2000 Chem. Rev. 100 2537

Pei J, Yu W-L, Haung W and Heeger A J 2000 Chem. Commun. 1631

Skotheim T A and Reynolds J R 1998 Handbook of conducting polymers: processing and application, CRC publication (Taylor and Francis group) 3rd ed. (New York: Marcel Dekker Inc)

Strukelji M, Papadimitrakooulous F, Miller T M and Rothberg L J 1995 Science 2671969

Stutzmann N, Friend R H and Sirringhaus H 2003 Science 299 188

Thompson B C et al 2005 New J. Chem. 291128

Udayakumar D and Adhikari A V 2006 Synth. Met. 1561168

Yang Chen-Jen and Jenekhe Samson A 1995 Macromol. 281180 